\title{
Discussion on Application of Molecular Biology Technology in Pathogenic Microorganism Examination
}

\author{
Zhang Lihua ${ }^{1}$ \\ ${ }^{1}$ ChiFeng Indusrty Vocational Technology College, Inner Mongolia, 024005 \\ (Remarks: The content of the article is about food inspection, food fermentation, food biotechnology, because the author is engaged in \\ food teaching)
}

\begin{abstract}
The implementation of pathogenic microorganism inspection work has a positive significance for the development of pharmaceuticals, food and other industries. It is precisely because of the existence of molecular biology technology that the reliability and accuracy of pathogenic microorganism inspection results can be better presented. This article summarizes the application advantages of molecular biology technology based on previous work experience. At the same time, this article discusses the application of molecular biology technology in pathogenic microorganism inspection from the application of polymerase chain reaction pathogenic microorganism inspection technology, biosensor pathogenic microorganism inspection technology, gene chip pathogenic microorganism inspection technology, accounting probe pathogenic microorganism inspection technology and PCR technology these five aspects.
\end{abstract}

\section{Introduction}

At present, with the continuous improvement of my country's medical technology, biological technology has also received many new development opportunities, which has provided great help for people to understand the world. People need to pay more attention to microbiological testing. With the gradual maturity of molecular biotechnology, the implementation strategy of pathogenic microorganism inspection work is becoming more and more perfect. At the same time, clinical microbiological testing technology can also be effectively improved and developed to better strengthen the level of microbiological testing.

\section{Analysis of the Advantages of Molecular Biology Technology}

Molecular biology technology is a basic discipline, the main research content is the structure and function of biological macromolecules and expression products, and the interaction between different structures is presented. At this stage, molecular biology technology represents a new pathogen detection method, which can further expand the detection range of pathogenic microorganisms and strengthen the accuracy and reliability of the detection results. It can also be seen from the actual microbiological testing process that the testing effect presented is extremely obvious, and this method has been approved by relevant researchers and has been fully applied in the fields of medicine, food and so on. As a highly scientific method for microbial detection, molecular biology technology can comprehensively detect microorganisms at the DNA and RNA levels. From the perspective of actual pathogenic microorganism inspection and detection, the most widely used technology at this stage is polymerase chain reaction technology, and also includes some biosensor technology, accounting probe technology and gene chip technology. The application of these technologies has also become more mature. It is also because of the role of these technologies that the detection time of pathogenic microorganisms has been greatly shortened, and the accuracy of experience is getting higher and higher. This can show a more positive role for microbial research and provide benefits for the implementation of subsequent research work condition.

\section{Materials and Methods}

\subsection{Normal Information}

In order to facilitate research, this article selected 50 specimens of a food inspection center between May 2017 and June 2019 as the research object. Among them, the number of food inspection specimens was 29 , and the number of food fermentation specimens was 21. From these specimen groups, we can understand that before this experiment was carried out, we obtained the approval of the relevant inspection department, and let every specimen understand the whole content of this experiment. After comparing the age and gender of the two groups of samples, there is no obvious difference, that is, the $\mathrm{P}$ value is greater than 0.05 , and a comparative study can be conducted. 


\subsection{Method}

Taking 50 specimens of a food inspection center between May 2017 and June 2019 as the research object to start collecting pathogenic microorganism inspection specimens. In general, each standard collects 2 copies of pathogenic microorganisms. We regard the No. 1 pathogenic microorganism test specimen as the first experimental group. The main detection method used is the conventional reverse transcription method, and the No. 2 pathogenic microorganism inspection standard is the second experimental group. The research method is the fluorescent quantitative reverse transcription method. In this way, we can clarify the rationality of the actual detection method by calculating the satisfaction rate of the two groups of pathogenic microorganism tests and the positive calculation rate of the pathogenic microorganism tests.

\subsection{Observation Index}

It can be seen that whether the satisfactory calculation rate of the pathogen microbiology test and the positive calculation of the pathogen microbiology test are in the

Table 1 Positive Calculation Rate of Pathogenic Microorganism Test of Two Groups of Specimens (n, \%)

\begin{tabular}{ccccc}
\hline Group & Number of Cases & Negative & Positive & Calculation Rate \\
\hline Group 1 & 50 & 23 & 27 & 54.0 \\
Group 2 & 50 & 11 & 39 & 785.0 \\
X $^{2}$ Value & & & & 6.417 \\
P Value & & & & 0.011 \\
\hline
\end{tabular}

\subsection{Comparison of the Calculation Rate of the Satisfaction of Pathogen Microbiological Examination between Two Groups}

It can be seen from the calculation results of the same state.

\subsection{Statistical Analysis}

The analysis software used in this experiment is SPSS21.0, the final count data is expressed as (\%), and the test index is $\mathrm{X}^{2}$. If $\mathrm{P}<0.05$, it proves that the entire study has statistical significance.

\section{Result Analysis}

\subsection{Comparison of the Positive Calculation Rate of Pathogenic Microorganism Test between Two Groups of Specimens}

From the analysis of the results of this experiment, it can be seen that the positive rate of pathogenic microorganism test presented by the 2 groups of samples is $78.0 \%$, which is much higher than the $54.0 \%$ of the 1 group. The difference is extremely obvious, $\mathrm{P}<0.05$, it has statistics significance of learning. It is shown in Table 1. pathogenic microorganisms in the experimental group 2 that the actual satisfaction rate is $94.0 \%$, which is significantly higher than the $74.0 \%$ in the experimental group 1. This also led to a more and more significant difference between the groups, with a $\mathrm{P}$ value below 0.05 , which is statistically significant, as shown in Table 2.

Table 2 Comparing the Calculation Rate of Pathogen Microbiological Examination Satisfaction between Two Groups of Specimens [n (\%)]

\begin{tabular}{cccrrrc}
\hline Group & $\begin{array}{c}\text { Number of } \\
\text { Cases }\end{array}$ & Dissatisfied & $\begin{array}{r}\text { Generally } \\
\text { Satisfied }\end{array}$ & $\begin{array}{r}\text { Relatively } \\
\text { Satisfied }\end{array}$ & $\begin{array}{c}\text { Very } \\
\text { Satisfied }\end{array}$ & $\begin{array}{c}\text { Calculation } \\
\text { Rate }\end{array}$ \\
\hline Group 1 & 50 & $13(26.0)$ & $15(30.0)$ & $10(20.0)$ & $12(24.0)$ & $37(74.0)$ \\
Group 2 & 50 & $3(6.0)$ & $16(32.0)$ & $13(26.0)$ & $18(36.0)$ & $47(94.0)$ \\
$X^{2}$ Value & & & & & & 7.440 \\
P Value & & & & & & 0.06 \\
\hline
\end{tabular}

\section{Discussion}

Up to now, my country's development in the field of life sciences has entered a new stage, and there has been a new turning point in life science research based on biological 
development of clinical testing for pathogenic microorganisms, molecular biology technology has been more widely used, which effectively compensates for the shortcomings of previous pathogenic microorganism testing.

It can also be seen from the research in the field of actual life sciences that polymerase chain reaction is one of the common molecular biology technical contents. When this technology is applied, it can synthesize DNA fragments, with the help of annealing, denaturation and other reactions, construct a reverse cycle process, and finally allow DNA to be truly amplified. In the traditional detection field, PCR has the following advantages: first, high sensitivity; second, obvious specificity; third, simple operation. It can also be seen from the clinical inspection work that PCR itself has high accuracy and can play an important role in microbiological inspection. Throughout the test results, the positive calculation rate of the pathogenic microorganism test in the experimental group 2 was $78.0 \%$, which was much higher than the $54.0 \%$ of the specimens in the experimental group 1. In addition, the satisfactory calculation rate of the pathogenic microorganism test in the experimental group 2 is $94.0 \%$, which is much higher than the $74.0 \%$ in the experimental group 1. It can also be seen from this that $\mathrm{P}<0.05$, this type of difference is statistically significant.

\section{Application of Molecular Biology Technology in Pathogenic Microorganism Examination}

\subsection{Polymerase Chain Reaction Pathogenic Microorganism Examination Technology}

With the continuous development of computer technology, the clinical pathogen microbial inspection operation gradually develops towards simplification and automation. For the application of molecular biology technology, it can also show the role of automated instruments in the detection of pathogenic microorganisms. More importantly, for the application of biochip technology, it can truly achieve pathogen detection, pathogen diagnosis and other operations, maximize the improvement of the traditional pathogen microbial inspection concept, while maintaining the final inspection quality, the pathogen microbial inspection efficiency Sexual and economic characteristics are presented. In contrast, the application of polymerase chain reaction technology is a common means of molecular biology technology in the detection of pathogenic microorganisms, and has a wide range of applications. With the continuous development of related technologies, new multiplex polymerase chain reaction has been derived, and fluorescence quantitative polymerase chain reaction technology has been implemented. It can be seen from the actual multiple polymerase chain reaction technology and application that it can be combined with a variety of specific primers under the same polymerase chain reaction state to promote the appearance of many DNA fragments on multiple different templates. In contrast, the application of the whole real- time fluorescence quantitative polymerase chain reaction technology is to concentrate the traditional polymerase chain reaction in fluorescence, and to realize comprehensive detection by means of fluorescence signals.

\subsection{Biosensor Pathogenic Microorganism Inspection Technology}

Throughout the application of the entire biosensor technology, it is mainly to effectively combine the advantages of molecular biology technology and sensor technology to construct a new pathogenic microbial inspection technology, which can also drive the progress of the entire microbial inspection field. In addition, with the gradual development of molecular biology technology, biosensor technology has become an important technical index content in molecular biology detection. For the understanding of biosensor technology, it can be defined as the fusion process of bio-derived materials and biobionic materials. Among them, the most common bioderived materials mainly include protein engineering, body-weight antibodies and so on. In contrast to biological activity materials, the main contents involved are proteins, antibodies, antigens and so on.

\subsection{Gene Chip Pathogenic Microorganism Inspection Technology}

Under the reasonable function of gene chip technology, it can combine multi-disciplinary knowledge such as computer science and biological first-level microelectronics. The main carriers are slides and nylon membranes, and a large amount of activity analysis is carried out by means of carrier per unit area. The density arrangement helps the staff to realize the effective inspection operation of various microorganisms. In general, through the application of gene chip technology, the actual microbial genetic information can be truly presented. Up to now, the actual gene sequence, pathogenic microorganism infection diagnosis and mutation mechanism research have been widely used in practical work. It can be seen from the comparison of traditional pathogen microbial inspection technology that for the application of gene chip technology, the infection between antibodies can be fully tested, which is also the basic process of locating pathogen types. At the same time, in the application process of gene chip technology, it can present higher test accuracy characteristics and determine the actual infection degree and infection range. In contrast, in the application process of gene chip technology, it can show the advantages of low demand and inspection automation, which is the root of its wide application. Since then, gene chip technology will get more opportunities for development, so that pathogenic microorganisms are on the same chip, truly accurate detection and analysis.

\subsection{Inspection Probe Pathogenic Microorganism Inspection Technology}

The implementation of the actual pathogenic microorganism inspection work, the application principle 
of nucleic acid probes is mainly based on basic complementation, especially in the calibration of DNA fragments, which can show the specificity and purpose of DNA hybridization, with the help of marker tracking to achieve a comprehensive inspection of pathogenic microorganisms. In addition, the application scope of the actual accounting probe technology is extremely wide. It is highly applicable to pathogenic microorganisms that cannot be observed and biochemically tested, and can also support those hepatitis viruses and epidemiological investigations. Comparing with the traditional pathogenic microorganism inspection technology, we can understand that the accounting probe technology can check the plant virus parasitic diseases. Relevant research shows that with the help of accounting probe technology, it can truly overcome the isotope labeling defects, shorten the half-life, and avoid more complicated operations.

\subsection{Application of PCR Technology}

It can also be seen from the execution of microbiological testing in the past that PCR technology is very common, and in the same reaction tube, it can show the specific advantages of different pathogenic microorganisms, and better complete the identification and detection of pathogenic microorganisms. Since the 1990s, my country has begun to apply PCR technology in the detection of infectious pathogenic microorganisms, such as human immunodeficiency virus, hepatitis virus. At this stage, PCR technology can also play a role in the detection of tuberculosis drug resistance genes and mycobacteria. When PCR technology is applied, it can show the characteristics of rapidity and sensitivity. As long as there are pathogens in the sample, PCR technology can achieve detection. More importantly, when PCR technology is applied, it will not be affected by the mixed specimens, and pathogens can be detected in the normal flora. However, it can be seen from the application of traditional PCR technology that there are also some problems. For example, when PCR detects RNA viruses, there are many detection steps involved, and some contaminants will also appear in the middle. Therefore, people need to avoid the defects of traditional PCR technology and gradually create new PCR technology.

\section{Conclusion}

In summary, molecular biology technology can show strong advantages in the detection of pathogenic microorganisms, and at the same time, it can show better detection reliability and accuracy characteristics, and establish a better application prospect for it. Therefore, in the follow-up development process, relevant departments can use the development of a single technology as a basic condition to achieve the combination of multiple inspection technologies to avoid the impact of pathogenic microorganism inspection efficiency.

\section{References}

1. Zhang Yue, Wang Jingyi. Application of molecular biology technology in microbiological examination of food [J]. Modern Food, 2020(01): 101-102.

2. Cao Lihua. Research on the application of molecular biology techniques in pathogenic microbiological testing $[\mathrm{J}]$. International Infectious Diseases (Electronic Edition), 2019, 8(03): 5-6.

3. Sun Wenlong, Guo Hong. Application of molecular biology technology in the detection of pathogenic microorganisms $[\mathrm{J}]$. China Urban and Rural Enterprise Health, 2019, 34(06): 46-47.

4. Li Ling, Yang Liwei. Analysis of the application effect of molecular biology technology in pathogenic microorganism testing $[\mathrm{J}]$. Journal of Clinical Laboratory Medicine (electronic version), 2019, 8(03): 173.

5. Liu Hua. Research progress in the application of molecular biology technology in microbiological testing $[\mathrm{J}]$. Electronic Journal of Clinical Medical Literature, 2019, 6(20): 197-198. 